\title{
A TECNOLOGIA EM SAÚDE: UMA PERSPECTIVA PSICOSSOCIOLÓGICA APLICADA AO CUIDADO DE ENFERMAGEM
}

\section{The technology in health: a psychossociological applied perspective to the care of nursing.}

\author{
La tecnología en salud: una perspectiva psicosociológica aplicada \\ al cuidado de enfermería.
}

Rafael Celestino da Silva ${ }^{1}$

Márcia de Assunção Ferreira ${ }^{2}$

\begin{abstract}
RESUMO
Objetiva-se demonstrar a relevância social do fenômeno tecnologia no cuidado em saúde como objeto de conhecimento psicossociológico. A tecnologia pode determinar o estilo de vida da sociedade, levando o homem a desenvolver sentimentos e comportamentos de adoração, medo, aproximação e afastamento. 0 fenômeno tecnologia em saúde integra pensamentos e discussões dos profissionais, fazendo circular informações entre eles, levando-os a elaborarem conhecimentos que alicerçam seus modos de agir. Pressupõe-se que a presença de recursos tecnológicos em ambientes de cuidado gera em quem cuida inquietações, sentimentos e comportamentos que se relacionam às representações sobre determinado objeto, no caso a tecnologia em saúde, as quais determinam diferentes modos de cuidar. Entender a tecnologia no cuidado em saúde na abordagem psicossociológica possibilita o conhecimento do campo que congrega elementos que constroem as representações sociais, no intento de compreender tais modos de cuidar.
\end{abstract}

Palavras-chave: Tecnologia Biomédica. Cuidados de Enfermagem. Enfermagem. Unidades de Terapia Intensiva.

\begin{abstract}
This study has a purpose of demonstrating the social relevance of technology as a phenomenon in the health care related to psychossociological knowledge. The technology can determine the way of life of the society, leading the man to develop feelings and behaviors of adoration, fear, approach and distancing. The phenomenon of the use of the technology in health, integrates thoughts and discussions, leading to circulation of the information among professionals; and then, producing knowledge as a foundation to the action of the health staff. The presence of technological resources in care environments generates restlessness, feelings and behaviors related to representations about an object, in this case, the technology in health. These representations produce different ways of care. The understanding of the technology in health care, based in a psychossociological approach, is useful to the knowledge of elements that builds social representations that are created to understand the ways of care.
\end{abstract}

Keywords: Biomedical Technology. Nursing care. Nursing. Intensive Care Units

\section{Resumen}

Se objetiva mostrar la relevancia social del fenómeno tecnología en el cuidado en salud como objeto de conocimiento psicosociológico. La tecnología puede determinar el estilo de vida de la sociedad, llevando al hombre a desarrollar sentimientos y comportamientos de adoración, miedo, aproximación y alejamiento. El fenómeno tecnología en salud integra pensamientos y discusiones de los profesionales, promoviendo la circulación de informaciones entre ellos, induciendolos a la elaboración de conocimientos que sirven de base a sus modos de actuar. Se presupone que la presencia de recursos tecnológicos en ambientes de cuidado genera en quien cuida inquietudes, sentimientos y comportamientos que se relacionan a las representaciones sobre determinado objeto, en este caso, la tecnología en salud, las cuales determinan diferentes maneras de cuidar. Entender la tecnología en el cuidado en la salud en el abordaje psicosociológico posibilita el conocimiento del campo que congrega elementos que construyen las representaciones sociales, con el objetivo de comprender tales maneras de cuidar.

Palabras clave: Tecnología Biomédica. Atención de Enfermería. Enfermería. Unidades de Terapia Intensiva.

${ }^{\dagger}$ Enfermeiro. Especialista em Enfermagem em Cardiologia e Médico-cirúrgica. Aluno do Curso de Doutorado da Escola de Enfermagem Anna Nery/EEAN - UFRJ. Membro do Núcleo de Pesquisa de Fundamentos do Cuidado de Enfermagem (Nuclearte). Brasil. E-mail: rafaenfer@yahoo.com.br, ${ }^{2}$ Doutora em Enfermagem. Membro do Núcleo de Pesquisa de Fundamentos do Cuidado de Enfermagem (Nuclearte). Professora Titular do Departamento de Enfermagem Fundamental da EEAN/UFRJ. Brasil. E-mail: marciadeaf@ibest.com.br 


\section{CONSIDERACÕES INICIAIS}

Este trabalho, de cunho teórico-conceitual, tem como objetivo trazer ao debate do campo da saúde a tecnologia aplicada ao cuidado às pessoas, principalmente no domínio da enfermagem de terapia intensiva, à luz de uma abordagem teórica específica que a constrói como fenômeno de Representação Social (RS). Antes de descrever melhor o problema que se apresenta na sustentação da relevância do debate proposto, destaca-se que o fenômeno em tela abarca o conceito de cuidado, situado no campo da enfermagem, e o de tecnologia, situado no campo da terapia intensiva. Na defesa de que a tecnologia no cuidado à saúde possa estar delimitada como objeto de conhecimento psicossociológico, o empenho inicial se faz no sentido de demonstrar a relevância e a "espessura social" deste fenômeno (objeto), que justificariam a existência de representações".

\section{A “ESPESSURA SOCIAL" DO FENÔMENO TECNOLOGIA NO CUIDADO EM SAÚDE}

A tecnologia pode influenciar e modificar nosso modo de vida e determinar influências em diversos campos tais como 0 social, o econômico e o ambiental. "A tecnologia é empregada para controlar, transformar ou criar coisas ou processos naturais ou sociais"2:31. Neste sentido, é capaz de determinar o estilo de vida de toda a sociedade, podendo, pois, ser entendida como "um parlamento de coisas no qual formas de civilização alternativas são debatidas e decididas"3:235. Com base na afirmação de Heidegger e Jaques Ellul, a tecnologia exerce controle cultural e social sobre o ser humano ${ }^{4}$.

Nos dias atuais, a(s) tecnologia(s) se faz(em) presente(s) em vários campos da atividade humana, e a humanidade encontra-se marcada pelos desafios políticos, sociais e econômicos decorrentes delas 5 . Em face disso, convém destacar que "frente à ciência e a tecnologia, o homem também parece estar adotando sentimentos e comportamentos biunívocos: adoração, medo, aproximação e afastamento"6:23.

Muitos são os afetos mobilizados diante do fenômeno Tecnologia, e, neste sentido, as influências e transformações geradas por ele na sociedade podem levar a posicionamentos, conflitos de opiniões, contradições, mudanças de valores e costumes nos sujeitos que estão inseridos no sistema social. Tais transformações acabam fazendo parte dos pensamentos e discussões daqueles que direta ou indiretamente convivem com as tecnologias e seus efeitos. Sendo assim, ao fazer parte dos conteúdos dos debates cotidianos dos sujeitos, a tecnologia acaba por tornar-se relevante e assunto em voga, seja nos grupos de amigos, na família ou nas conversas informais com desconhecidos.

A circulação de informações sobre os mais novos feitos da ciência no lançamento de inovaç̃̃es tecnológicas e seus modos de atuar no curso da vida dos sujeitos, e, ainda, os impactos que isto traz para quem delas usufrui ou não, por falta de acesso ou opção, pode levar os grupos a elaborarem conhecimentos que irão alicerçar seus modos de agir, justificando suas condutas diante dela - a tecnologia. Neste sentido, convém trazer à tona que os fenômenos sociais que nos permitem identificar de maneira concreta as representações e de trabalhar sobre elas são as conversações, dentro das quais se elaboram os saberes populares e o senso comum? ${ }^{7}$.

\section{Focalizando o objeto: repercussões dos usos da tecnologia no trabalho do enfermeiro e no cuida- do de enfermagem}

Se a tecnologia trouxe, e ainda traz, um impacto importante com mudanças significativas para o mundo do trabalho de um modo geral, este pode ser sentido, também, em especial, no trabalho da enfermagem, principalmente no âmbito de setores de cuidado intensivo.

Progressivamente, as mudanças se fizeram sentir na infraestrutura de cuidados e atendimentos hospitalares6, pois os serviços de saúde passaram a dispor de técnicas, instrumentos, métodos e matérias-primas diversos, o que implicou não só a reconfiguração de espaço físico e de atuação profissional, mas em novas modalidades de prevenção, diagnóstico e tratamento dos problemas de saúde.

Assim, a incorporação das tecnologias de ponta nos cenários de assistência implicou um redimensionamento do espaço do cuidado, com repercussões diretas no trabalho dos enfermeiros, na medida em que traz mais uma atribuição para estes que, além de cuidarem do cliente, devem controlar a(s) máquina(s) e todo 0 arsenal de equipamentos e artefatos inerentes a determinadas terapias. Então, embora pensemos que a tecnologia deva ser utilizada na prática hospitalar como uma ferramenta auxiliar no cuidado de enfermagem, que supre as necessidades crescentes do aumento da complexidade do cuidado oferecido pelas enfermeiras e possibilita a prestação de um cuidado de qualidade, com obtenção de maior eficácia, minimizando o tempo gasto na realização dos procedimentos, por outro lado, é preciso pensar sobre os efeitos que 0 avanço tecnológico remete 8 .

A incorporação das novas tecnologias vem imprimindo mudanças gradativas nos cuidados prestados pelo enfermeiro. Isso, por vezes, gera medo e angústia para alguns, enquanto para outros representa status, incentivo à busca de aperfeiçoamento técnico-científico, determinando condutas ambivalentes de maior proximidade ou afastamento do cliente. Enfim, o fenômeno tecnologia em saúde, pela complexidade e demandas diferenciadas que por vezes traz no manejo do cuidado ao cliente, faz com que os profissionais pensem, reflitam, discutam e troquem experiências sobre o cuidado ao cliente em um ambiente tecnológico.

De maneira particular e influenciado por toda a estrutura organizacional peculiar de uma instituição de saúde e, nesta, a do cenário da Terapia Intensiva, o enfermeiro que lida diariamente com o cuidado aos clientes, quando necessita manejar uma gama de aparatos tecnológicos que medeia a 
assistência por ele a ser prestada, acaba por comunicar condutas ou modos de agir diferenciados que se relacionam a uma série de quesitos afeitos ao seu tempo de formação, à sua prática (se especializada ou não), às suas preferências pessoais por trabalhar em um outro setor/clientela, entre outros. Tudo isso contribui para a forma com que o enfermeiro irá construir as suas representações sobre a tecnologia e a aplicação desta no cuidado ao cliente.

As enfermeiras novatas, por exemplo, apresentam sentimentos, frustrações, expectativas que as acompanham e interferem em seu modo de agir, pensar e reagir diante das situações cotidianas da prática da enfermagem, sendo necessário um espaço para se discutir os limites e dificuldades, sentimentos e reações advindas da prática ${ }^{9}$. Tal afirmação vem sustentar a idéia de que os enfermeiros constroem representações acerca de objetos afeitos ao ambiente tecnológico de cuidado, principalmente quando as situações vividas thes parecem estranhas, como é o caso do desconhecimento do manejo e/ou emprego de algum aparato tecnológico ao cuidado. Ainda mais, quando se trata de enfermeiros iniciantes ou novatos, cada encontro se apresenta como uma situação nova, o que gera, para quem cuida, uma sensação semelhante à de um estreante.

É fato que as novas tecnologias modificam o cotidiano das pessoas, seja no âmbito laboral ou fora dele. Inclusive, devemse levar em conta as reconfigurações das relações interpessoais diante do fenômeno das incorporações tecnológicas no dia-adia das pessoas, que, sem dúvida, sob muitos aspectos, altera o ritmo de vida e de trabalho.

É com base nessas aproximaç̃es primeiras ao objeto e no entendimento de que as representações sociais são "alguma coisa que emerge das práticas em vigor na sociedade e na cultura que as alimenta, perpetuando-as ou contribuindo para a sua própria transformação" ":40, que se reúnem os argumentos na defesa de que a tecnologia em saúde pode ser configurada como objeto de conhecimento psicossociológico. Acrescentase a isso que a "espessura" de um objeto pode ser traduzida da seguinte maneira: 0 objeto em questão "se encontra implicado, de forma consistente, em alguma prática do grupo, aí incluída a da conversação, a da exposição aos meios de comunicação de massa"1:42.

Embora haja um número ascendente de pesquisas conduzidas sob a ótica da teoria das representações sociais, não há unanimidade entre os pesquisadores acerca dos critérios para definição das representações sociais. Considerar a gênese social das representações sociais significa adotar alguns critérios de diagnóstico que possibilitem caracterizar as representações além de sua estrutura interna e do simples compartilhamento de pensamentos e idéias ${ }^{10}$. Entre esses critérios, destacam-se o de relevância, pois as representações sociais se referem apenas a objetos ou questões socialmente relevantes, ou seja, é necessário que o padrão de comportamento dos indivíduos ou grupos mude em presença desses objetos para que eles sejam considerados relevantes, gerando um trabalho representacional por parte dos atores sociais ${ }^{11}$; e o critério de prática, no qual só se deve falar do surgimento de uma representação social num grupo se pensamento e ação dos seus membros forem modificados em função do surgimento desta representação, isto é, pensamento e ação não podem ser concebidos separadamente quando se fala em representação social, mas sim devem ser vistos como epistemologicamente equivalentes ${ }^{12}$.

Nesse sentido, tomando como referência os critérios para diagnóstico das representações sociais, bem como a fundamentação teórica apresentada, pensamos que a tecnologia aplicada no cuidado em saúde se constitui num fenômeno que leva a uma prática representacional pelos atores sociais, neste caso os enfermeiros, na medida em que a sua incorporação em ambientes tecnológicos de cuidado provoca um pensar e um agir relacionados ao modo como se constrói o conhecimento em relação a esta questão, bem como a forma como se experienciam e vivenciam as mudanças no cotidiano de trabalho.

No que tange ao conhecimento, o ritmo impresso pelas novas tecnologias nos processos de atualização e desatualização do saber "é cada vez mais rápido, acrescentando novos componentes à questão da sobrevivência deste saber" ${ }^{16: 74}$. Sendo assim, considerar a tecnologia enquanto fenômeno psicossocial, situado no contexto da saúde e, em especial, do cuidado de enfermagem, significa trazer à tona o que os profissionais pensam e como agem diante da incorporação das novas tecnologias no contexto do cuidado, bem como as mudanças que ocorrem quanto à atuação profissional por conta desta incorporação.

\section{ENTENDENDO OS PROCESSOS DE CUIDAR EM ENFERMAGEM NOS AMBIENTES TECNOLÓGICOS A PARTIR DE UMA ABORDA- GEM PSICOSSOCIOLÓGICA}

A partir das considerações expostas até então, torna-se evidente que as tecnologias já presentes em ambientes de cuidado, bem como aquelas que são incorporadas no intuito de contribuir com a recuperação dos clientes, interferem sobremaneira no modo como os enfermeiros desenvolvem os cuidados aos sujeitos sob sua responsabilidade, ou seja, interferem nas ações e comportamentos que são realizados no sentido de favorecer, manter ou melhorar a condição humana no processo de viver ou morrer; em última instância, interferem no processo de cuidar.

0 processo de cuidar é um processo interativo, que envolve um movimento no sentido de conduzir a transformação, entendido nas dimensões física, psicológica, social, ambiental e cultural $^{13}$. Cuidar, então, compreende aspectos pessoais e sociais, sensibilidade, respeito, solidariedade, interesse. Implica, portanto, colocar-se no lugar do outro, interagir e responsabilizar-se com o outro no intento de aliviar seu sofrimento ou proporcionar seu bem-estar ${ }^{14}$. 
No processo de cuidar, a relação interpessoal proporciona uma assistência baseada no respeito das individualidades, na comunicação e relacionamento entre as pessoas, expressandose através de gestos de amor, do ouvir, do não-verbal, da observação, confiança e afeto ${ }^{15}$.

No entanto, o cuidado adquire diferentes formas conforme o significado das ações de saúde para cada ser humano, ou seja, os modos de cuidar dos enfermeiros são orientados por um conjunto de idéias teórico-filosóficas, as quais justificam a adoção de determinadas condutas. Assim, os modos de cuidar em enfermagem não devem ser entendidos dentro de um contexto de pretensa neutralidade, mas a partir de sua dimensão político-social e sua implicação sobre a vida dos cidadãos ${ }^{14}$. Neste sentido, pensar sobre os modelos de cuidado em enfermagem remete ao entendimento do referencial teóricofilosófico em que se fundam, das reflexões pessoais e coletivas envolvidas, perpassando pela compreensão do valor desse cuidado no contexto sócio-coletivo em que se insere ${ }^{14}$.

Considerar então a compreensão psicossociológica dos modos de cuidar dos enfermeiros em cenários tecnológicos é afirmar que tais modos são construídos a partir do sentido e do significado que é atribuído à tecnologia, isto é, o indivíduo orienta suas ações a partir da codificação que ele faz da realidade, da forma como pensa sobre as coisas reais e imaginárias do seu mundo que o faz reconstituir a realidade com a qual se confronta.

A observação de tais modos de cuidar vem demonstrando dificuldades para utilização dos recursos tecnológicos disponíveis como instrumentos para a identificação e o atendimento das necessidades dos clientes que estejam sob cuidados nestes ambientes. Isso pode ser manifestado no campo dos cuidados instrumentais, seja por um negligenciamento de cuidados, em virtude de uma falta de conhecimento que gera medo na manipulação de equipamentos e máquinas, seja por um afastamento do cliente, no sentido de não-envolvimento em situações que exijam experiência e conhecimento para proceder ao encaminhamento e resolutividade dos problemas que porventura venham a acontecer.

No campo dos cuidados expressivos, observa-se atualmente que os profissionais de enfermagem supervalorizam 0 conhecimento sobre os aparatos tecnológicos, o qual representa statuse poder, e, em contrapartida, desconhecem sobre a pessoa que estão cuidando, foco principal dos cuidados de enfermagem. As mudanças tecnológicas alteraram o perfil da enfermeira, que passa a ter um saber tecnificado por meio da apropriação da ciência, o qual pode estar levando-a a desenvolver uma prática excessivamente centrada no diagnóstico e na intervenção, na qual o ser humano é traduzido apenas como informaçãa ${ }^{6}$.

Os processos de cuidar em enfermagem estão assumindo, assim, características próprias, as quais dizem respeito aos sujeitos que conduzem a ação (cuidado), o profissional. Nesse sentido, os sujeitos compreendem e interpretam diferentemente a situação na qual se encontram, e não se comportam de maneira semelhante diante de um procedimento que permanece idêntico. Considera-se, portanto, 0 ato de representação um ato de pensamento pelo qual o sujeito se relaciona com um objeto, um processo de elaboração cognitiva e simbólica que vai orientar os comportamentos.

Entender então os processos de cuidar a partir de uma abordagem psicossociológica remete à estreita relação a saber: o enfermeiro cuida do cliente portador de inúmeros aparatos tecnológicos a partir da representação que ele faz desta tecnologia, que por sua vez implica a possibilidade de conhecer como se constrói tal representação, e, consequentemente, intervir sobre fatores ou elementos que a constituem, na dependência das repercussões que tal representação traz ao cliente.

Portanto, a compreensão dos modos de cuidar dos enfermeiros em ambientes tecnológicos sob a ótica da psicossociologia aponta para a possibilidade de identificar os fatores que interferem na construção da representação dos enfermeiros sobre a tecnologia enquanto fenômeno social, que, por sua vez, justificam seus modos de cuidar. As possíveis contribuições disso são as possibilidades de reflexão sobre a atuação do profissional e o cuidado por ele prestado, trazendo à tona uma discussão que envolve qualificação profissional, formação especializada, qualidade da assistência, entre outras.

\section{CONSIDERAÇÕES FINAIS}

A tecnologia no cuidado desperta nos enfermeiros fascínio, medo, estímulo ao saber e outras sensações semelhantes às de iniciantes ou novatos diante de situações novas e estranhas, algo inusitadas.

No intento de conformação desta idéia e de sustentação de uma linha argumentativa em face do fenômeno posto em discussão, reitera-se o pressuposto teórico de que a presença constante de novos recursos tecnológicos em ambientes de cuidado avançado gera em quem cuida inquietações, sentimentos e comportamentos que se relacionam à existência de representações sobre um determinado objeto, no caso a tecnologia em saúde. Por isso, tal objeto pode ser pensado à luz da Teoria das Representações Sociais, uma vez que as representações sociais referem-se a objetos ou questões socialmente relevantes ${ }^{10}$, e tal relevância se assenta nas mudanças de padrão de comportamento de indivíduos ou grupos diante da presença do mesmo ${ }^{11}$.

Logo, para o campo da saúde, e em especial do cuidado de enfermagem, importa conhecer como o enfermeiro vem enfrentando as transformações que se colocam no cotidiano da prática de cuidar, à luz das tecnologias cotidianamente incorporadas, e de que forma isso implica nas suas atitudes e modos de agir. No fim, importa saber o impacto que isso traz para a prestação do cuidado integral e de qualidade à clientela. 


\section{REFERÊNCIAS}

1- Sá CP. Sur les relations entre représentations sociales, pratiques sócio-culturelles et comportement. Papers on Social Representations $1994 ; 3(1): 40-46$.

2- Sancho JM. A tecnologia: um modo de transformar um mundo carregado de ambivalência. In: Sancho JM. Para uma tecnologia educacional. Porto Alegre(RS): Artmed; 1998.

3- Feenberg A. Critical theory of technology. New York(USA): Oxford University Press; 1991.

4 Feenberg A. Questioning technology. London (UK): Routeledge; 1999.

5- Domingues D. A humanização das tecnologias pela arte. In: Domingues D, organizador. A arte no século XXI: a humanização das tecnologias. São Paulo(SP): Ed UNESP; 1997.

6- Laino A. Mudanças técnico científicas hospitalares, trabalho e sociedade: estudo de caso na Itália. Niterói(RJ): EDUFF; 1996.

7- Moscovici S. Prefácio. In: Guareschi PA, Jovchelovitch S, organizadores. Textos em representações sociais. $2^{\mathrm{a}}$ ed. Petrópolis (RJ): Vozes; 1995

8- Barbosa SFF. A transcendência do emaranhado tecnológico em cuidados intensivos: a (re)invenção possível. Blumenau(SC): Nova Letra; 1999.
9- Espírito Santo FH. Saberes e fazeres de enfermeiras novatas e veteranas sobre o cuidado de enfermagem no cenário hospitalar [tese de doutorado]. Rio de Janeiro (RJ): Escola de Enfermagem Anna Nery/UFRJ; 2003.

10- Wagner W. Sócio-gênese e características das representações sociais. In: Moreira ASP, Oliveira DC, organizadoras. Estudos interdisciplinares de representação social. Goiânia(GO): AB; 1998.

11- Sperber D, Wilson D. Relevance- communication and cognition. Oxford (USA): Blackwell; 1986.

12- Himmelweit HT. Societal psychology: implications and scope. In: Himmelweit HT, Gaskell G, editores. Societal psychology. London(UK): Sage; 1990.

13- Waldow VR. Examinando o conhecimento na enfermagem. In: Meyer DE, Waldow VR, Lopes MJM. Marcas da diversidade: saberes e fazeres da enfermagem contemporânea. Porto Alegre(RS): Artmed; 1998.

14- Souza ML, Sartor VVB, Padilha MICS, Prado ML. 0 cuidado de enfermagem: uma aproximação teórica. Texto\& Contexto Enferm 2005 abr/jun; 14(2): 266-70.

15- Wolff LR. Representações sociais de mulheres sobre assistência no trabalho de parto e parto [tese de doutorado]. Rio de Janeiro (RJ): Escola de Enfermagem Anna Nery/UFRJ; 2004. 